IN PRACTICE

\title{
Improvement in the clinical cure rate of outpatient management of pelvic inflammatory disease following a change in therapy
}

\section{A Piyadigamage, J Wilson}

Sex Transm Infect 2005;81:233-235. doi: 10.1136/sti.2004.012377

See end of article for authors' affiliations

....................

Correspondence to: A Piyadigamage, Department of

Genitourinary Medicine, The General Infirmary at Leeds, Leeds LS1 3EX, UK; anura.piyadigamage@ chesterfieldroyal.nhs.uk

Accepted for publication 9 September 2004 Objective: In the United Kingdom many genitourinary medicine clinics use oral doxycycline and
metronidazole to treat pelvic inflammatory disease (PID). A retrospective case note review of PID treatment at our department in 2000 showed that the clinical cure rate (CCR) was only $55 \%$ with oral doxycycline and metronidazole for 2 weeks. We therefore added ceftriaxone $250 \mathrm{mg}$ intramuscularly to the doxycycline and metronidazole for treating PID. We have repeated the review and compared the results with those from 2000.

Methods: All patients diagnosed as having PID between 1 July 2002 and 31 December 2002 were identified. These episodes were diagnosed on clinical presentations of pelvic pain, vaginal discharge or bleeding, and cervical motion tenderness on physical examination. The CCR was defined as patients who fully resolved their symptoms and signs during 2 week and 4 week follow up. The results were compared with those from 2000.

Results: Women receiving ceftriaxone, doxycycline, and metronidazole had a CCR of $72 \%$. In 2000 the CCR for women receiving only doxycycline and metronidazole was $55 \%$. There were only $8 \%$ nonresponders in 2002 compared with $18 \%$ in 2000. Comparing CCR and non-response rate, in 2002 there was a significant improvement in cure rate, $O R 3.01(95 \% \mathrm{Cl} 1.28$ to 7.47$) \mathrm{p}=0.009$. Using an intent to treat analysis and including the defaulters as treatment failures there was still a significant improvement in cure rate, OR 2.03 (95\% Cl 1.18 to 3.50$) \mathrm{p}=0.009$.

Conclusions: The treatment of PID with ceftriaxone, doxycycline, and metronidazole gave a significantly higher CCR than doxycycline and metronidazole. Our experience would suggest that doxycycline and metronidazole alone is not a suitable regimen for treatment of PID in the United Kingdom.
W omen with pelvic inflammatory disease (PID) frequently present to genitourinary medicine (GUM) clinics. The UK national guidelines recommend treatment with a single parenteral third generation cephalosporin, followed by oral doxycycline and metronidazole for 2 weeks, or oral ofloxacin and metronidazole for 2 weeks. ${ }^{1}$ However, within GUM clinics throughout the United Kingdom there has been some controversy about what is the best treatment for the management of PID in such an outpatient setting. A survey conducted in 2001 suggested that $71 \%$ of clinics in the United Kingdom still used a combination of just oral doxycycline and metronidazole to treat PID even though there is little published evidence to support such a regimen. ${ }^{2}$ That had been the treatment used in this clinic until a retrospective case note review of outpatient management of PID, in 2000, showed that only $55 \%$ of patients achieved clinical cure with oral doxycycline and metronidazole when given for 2-4 weeks. ${ }^{3}$ Following these results the clinic treatment guidelines for PID were changed by adding ceftriaxone $250 \mathrm{mg}$ by intramuscular injection to the oral doxycycline and metronidazole. This treatment was chosen after considering the published clinical evidence, the background prevalence of infections associated with PID, and local antibiotic sensitivity patterns. ${ }^{145}$ The ciprofloxacin resistance rate within our department was $15 \%$ in 2000.

The new treatment protocol was introduced on 1 July 2001. As it usually takes some time for any change in practice to be fully effective, the case note review was repeated 1 year after switching to this new treatment regimen. This paper presents the results of the new regimen and compares these with the results from the previous review in $2000{ }^{3}$

\section{METHODS}

All the patients diagnosed as having PID in our outpatient clinic between I July 2002 and 31 December 2002 were identified using the computerised clinic coding system. The diagnoses had been made on clinical presentations with lower abdominal pain, plus vaginal discharge, or postcoital bleeding, or intermenstrual bleeding, and either cervical motion tenderness and/or uterine/adnexal tenderness on bimanual examination, and a negative pregnancy test. Case notes were reviewed retrospectively by the authors and the following data were recorded: a positive urethral and/or endocervical culture of Neisseria gonorrhoeae; positive polymerase chain reaction (PCR) for Chlamydia trachomatis; bacterial vaginosis on Gram stain; antibiotics prescribed; clinical cure at follow up visit; microbiological cure at follow up; partner notification and treatment. The clinical cure was defined as patients whose symptoms and signs had resolved at the 2 week or 4 week follow up appointment. Defaulters and non-responders were also identified. Non-responders were defined as patients who continued to complained of lower abdominal pain with either cervical motion tenderness and/or uterine/adnexal tenderness on bimanual examination at 2 weeks and 4 weeks after treatment.

Abbreviations: CCR, clinical cure rate; GUM, genitourinary medicine; PID, pelvic inflammatory disease 
Table 1 The antibiotic regimens prescribed, and the clinical response

\begin{tabular}{lcccc}
\hline Drug regimen & $\begin{array}{l}\text { No of } \\
\text { patients }\end{array}$ & $\begin{array}{l}\text { No with } \\
\text { clinical cure }\end{array}$ & $\begin{array}{l}\text { No who } \\
\text { defaulted } \\
\text { follow up }\end{array}$ & $\begin{array}{l}\text { No who did not } \\
\text { respond to treatment }\end{array}$ \\
\hline $\begin{array}{l}\text { Ceftriaxone + doxycycline + } \\
\text { metronidazole }\end{array}$ & 131 & $94(72 \%)$ & $27(20 \%)$ & $10(8 \%)$ \\
$\begin{array}{l}\text { Ofloxacin + metronidazole } \\
\text { Ciprofloxacin + doxycycline + }\end{array}$ & 7 & 2 & 2 & 3 \\
metronidazole & 4 & 1 & 1 & 2 \\
$\begin{array}{l}\text { Azithromycin + metronidazole } \\
\text { Erythromycin + metronidazole }\end{array}$ & 3 & 2 & 1 & 0 \\
Total & 147 & 1 & 0 & 1 \\
\hline & & 100 & 31 & 16 \\
\hline
\end{tabular}

The data were compared with the previous results collected during the same months in 2000. Statistical analysis was by $\chi^{2}$ tests.

\section{RESULTS}

In all, 147 women were diagnosed as having PID during this period in 2002. Chlamydia and gonorrhoea were identified in $38(26 \%)$ and $18(12 \%)$ cases, respectively, with six $(4 \%)$ having both infections. Bacterial vaginosis was diagnosed in $49(33 \%)$ cases. The figures for 2000 were chlamydia in 46 $(30 \%)$, gonorrhoea in six $(4 \%)$, both infections in three $(2 \%)$, and bacterial vaginosis in $35(23 \%)$. The increase in the proportion of women with gonorrhoea between 2002 and 2000 was statistically significant, OR 3.35 (95\% CI 1.22 to 10.59), $\mathrm{p}=0.01$. All women with gonorrhoea and/or chlamydia at presentation had microbiological cure at follow up.

Five different antibiotic regimens were used in 2002 even though only two different regimens are recommended in the clinic guidelines. Only $89 \%$ of the women received the clinic recommended treatment. Details of the antibiotic regimens prescribed, and the clinical response are shown in table 1.

In women who received the first line recommended treatment of ceftriaxone $250 \mathrm{mg}$ intramuscular single dose followed by a 2 week course of doxycycline $100 \mathrm{mg}$ and metronidazole $400 \mathrm{mg}$ twice daily, the clinical cure rate (CCR) was $72 \%$ in this review. In 2000 the CCR for women receiving doxycycline and metronidazole was 55\%. There were only $8 \%$ non-responders in 2002 compared with $18 \%$ in 2000. The comparisons between the two reviews are shown in table 2. There was a significant improvement in CCR in 2002 compared with 2000, OR 3.01 (95\% CI 1.28 to 7.23 ) p $=0.009$. Using an intent to treat analysis, and including defaulters as treatment failures, there was still a significant improvement in CCR, OR 2.03 (95\% CI 1.18 to 3.50 ) p $=0.009$.

No adverse reactions were experienced to the ceftriaxone injections, and during this 6 month period only two patients refused intramuscular treatment. However, one disadvantage of the current regimen was the extra nursing time that was required to administer an intramuscular injection rather than dispensing just oral medication. This extra time equated to 10 more minutes of nursing time per patient treated.
Partner notification was discussed and recorded in the notes in $86 \%$ (126/147) of the patients; however, only 56\% $(82 / 147)$ of partners were actually seen in the clinic and treated. In 2000 partner notification was discussed in $87 \%$ $(131 / 150)$, but $70 \%(106 / 150)$ of male partners were treated. This is a significant drop in proportion of male partners treated, OR 0.52 (95\% CI 0.32 to 0.87 ), p $=0.01$. However, in 2002 the partner notification rate was higher among the women found to have gonorrhoea or chlamydia (49/ $51=96 \%)$, with $76 \%(39 / 51)$ of their contacts seen and treated; these figures are not significantly different from 2000 .

\section{DISCUSSION}

This study demonstrates that by adding ceftriaxone to doxycycline and metronidazole, for the outpatient treatment of PID, a significantly higher CCR was achieved than with doxycycline and metronidazole alone. The ceftriaxone injections were well tolerated, but required extra nursing time for their administration. However, this extra nursing time was more than compensated for by the improvement in CCR that meant that fewer follow up visits were required.

There was a significant increase in the proportion of cases with gonorrhoea between 2000 and 2002. This was in keeping with the national increase in gonorrhoea throughout the United Kingdom during this time. ${ }^{6}$ This increase in gonorrhoea reinforces the need for an effective antigonococcal treatment within any PID regimen. This region has high levels of quinolone resistant gonorrhoea, meaning that a third generation cephalosporin is the first line treatment, so this needs to be included in treatment for PID.

The diagnosis of PID was made on clinical grounds, and this is one of the limitations of this study as individual clinical judgment may vary. This particularly applies to the comparisons of results between 2000 and 2002. However, the same protocol was used during the two time periods; all the patients had presented with recent onset of lower abdominal pain, with vaginal discharge or post coital bleeding or inter menstrual bleeding, and they all had cervical motion tenderness and/or uterine/adnexal tenderness on bimanual examination. Also, most of the doctors examining the

Table 2 The comparison of the results between 2000 and 2002

\begin{tabular}{llllll}
\hline Year & Drug regimen & $\begin{array}{l}\text { No of } \\
\text { patients }\end{array}$ & $\begin{array}{l}\text { No with } \\
\text { clinical cure }\end{array}$ & $\begin{array}{l}\text { No who } \\
\text { defaulted follow up }\end{array}$ & $\begin{array}{l}\text { No who did not } \\
\text { respond to treatment }\end{array}$ \\
\hline 2000 & $\begin{array}{l}\text { Doxycycline }+ \\
\text { metronidazole }\end{array}$ & 135 & $75(55 \%)$ & $36(27 \%)$ & $24(18 \%)$ \\
\hline $\begin{array}{l}\text { Ceftriaxone }+ \\
\text { doxycycline }+ \\
\text { metronidazole }\end{array}$ & 131 & $94(72 \%)$ & $27(20 \%)$ & $10(8 \%)$ \\
\hline
\end{tabular}




\section{Key messages}

- The clinical cure rate of pelvic inflammatory disease (PID) is low with a combination of oral doxycycline and metronidazole alone

- There has been a significant increase in rates of gonococcal PID and quinolone resistant gonorrhoea

- Adding a single parenteral dose of ceftriaxone to doxycycline and metronidazole achieved a significantly higher clinical cure rate

- Doxycycline and metronidazole alone is not a suitable regimen for treatment of PID in the United Kingdom

patients and making the diagnoses were the same in the two reviews.

Another weakness of this study is that the reviews were conducted 2 years apart even though the same 6 month period of each year was used, so it is possible that we were comparing different patient populations. The findings from these retrospective case note reviews are obviously much less robust than a direct comparison of the treatments using a randomised controlled trial (RCT); however, to conduct a RCT comparing these treatments would have been much more costly, and less feasible.

There was a significant reduction in male partners seen and treated between 2000 and 2002, although the figures for partner notification in women with gonorrhoea and chlamydia were not different. In view of the general rise in STI diagnoses in the clinic between 2000 and 2002 there had been a change in policy regarding health adviser partner notification of PID. In 2002 all patients with a diagnosis of PID were still seen at first visit by a health adviser but only those with gonorrhoea and chlamydia were seen again at follow up if partner notification had not been completed. This may explain the difference in partner notification figures.

A study conducted in the United States showed that outpatient treatment with single dose parenteral cefoxitin followed by oral doxycycline was as effective as inpatient intravenous cefoxitin and doxycycline, followed by oral doxycycline, in mild to moderate PID. ${ }^{7}$ Our study demonstrates that the outpatient treatment of PID with ceftriaxone, doxycycline, and metronidazole gave a significantly higher CCR than doxycycline and metronidazole alone. These results, plus the increasing rates of gonorrhoea and the increase in quinolone resistance throughout the United Kingdom, would suggest that doxycycline and metronidazole alone is not a suitable regimen for treatment of PID in the United Kingdom. ${ }^{8}$

\section{CONTRIBUTORS}

Both authors designed the study; AP collected the data and JDW performed the data analysis; both authors wrote the manuscript.

\section{Authors' affiliations}

A Piyadigamage, J Wilson, Department of Genitourinary Medicine, The General Infirmary at Leeds, Leeds LS1 3EX, UK

Conflict of interest: none.

\section{REFERENCES}

1 Ross JDC. UK national guidelines for the management of pelvic inflammatory disease. Sex Transm Infect 1999;75:S54-6.

2 Riddle LA, Rooney G. The treatment of out patient pelvic inflammatory disease (PID) in GUM clinics - a national survey. MSSVD spring meeting 2001, abstract 35 .

3 Piyadigamage A, Wilson JD. An audit of outpatient management of pelvic inflammatory disease. Int J STD AIDS 2002;13:577-9.

4 Arredondo JL, Diaz V, Gaitan H, et al. Oral clindamycin and ciprofloxacin versus intramuscular ceftriaxone and oral doxycycline in the treatment of acute mild to moderate pelvic inflammatory disease in outpatients. Clin Infect Dis 1997;24:170-8.

5 GRASP (The Gonococcal Resistance to Antimicrobial Surveillance Programme). Annual report 2002.

6 Health Protection Agency, SCIEH, ISD, National Public Health Service for Wales, CDSC Northern Ireland and the UASSG. Renewing the focus. HIV and other sexually transmitted infections in the United Kingdom in 2002. London: Health Protection Agency, November, 2003.

7 Ness RB, Soper DE, Holley RL, et al. Effectiveness of inpatient and outpatient treatment strategies for women with pelvic inflammatory disease: results from the Pelvic Inflammatory Disease Evaluation and Clinical Health (PEACH) randomised trial. Am J Obstet Gynecol 2002;186:929-37.

8 Ross JDC. Extracts from "clinical evidence": pelvic inflammatory disease. BMJ $2001 ; 322: 658-9$. 